\title{
The Atmospheric Scanning Electron Microscope (ASEM) Observes the Critical Moment of Platelet Generation from Megakaryocytes in Solution
}

\author{
Chikara Sato ${ }^{1}$, Hidetoshi Nishiyama ${ }^{2}$, Yuusuke Maruyama ${ }^{1}$, Tatsuhiko Ebihara ${ }^{1}$, Mitsuo Suga ${ }^{2}$, \\ Masayuki Yamamoto ${ }^{3}$ and Hozumi Motohashi ${ }^{3}$ \\ 1. National Institute of Industrial Science and Technology (AIST), Tsukuba, 305-8566, Japan \\ 2. JEOL Ltd., 1-2 Musashino 3-chome, Akishima, Tokyo 196-8558, Japan \\ 3. School of Medicine, Tohoku University, 2-1 Seiryo-cho, Sendai 980-8575, Japan
}

The new Atmospheric Scanning Electron Microscope (ASEM) is Correlative Light-Electron Microscope (CLEM), in which the optical microscope (OM) and SEM quasi-simultaneously observe samples in water solution under open atmosphere [1]. In the system, an inverted SEM observes the wet sample from beneath an open dish while an optical microscope (OM) observes it from above (Figure 1). The disposable dish with an electron transparent $\mathrm{SiN}$ film window can hold a few milliliters of culture medium, and allows various types of cells to be cultured in a stable environment, e.g., a $\mathrm{CO}_{2}$ incubator. The film with poly-L-lysine coating allows primary megakaryocytes (MKs) to be cultured and extend proplatelets (Figure 1).

Proplatelets are beaded cell projections generated from mature megakaryocytes during the production of platelets, which are indispensable cellular components of blood for hemostasis. MKs extend plump pseudopodia that give rise to long branched proplatelets, from which platelets are shed off. The precise mechanisms of their generation are not fully understood, for example, whether proplatelets detach from MKs in bulk or in pairs. This is mainly because platelets are too small to be observed using OM. We cultured MKs on ASEM dish and fixed the cells at an appropriate timing captured with OM. The cells were stained with heavy metal solution and observed at high resolution with the inverted SEM. The pseudopodia extended beaded strings with internal vesicles, necessary for blood clot formation, which is related to cerebral or myocardial infarction under pathological conditions.

ASEM also realized high throughput immune-EM in solution, and visualized molecular complex formation of signaling molecules, in response to $\mathrm{Ca}^{2+}$ store depletion in endoplasmic reticulum (ER). STIM1 is a $\mathrm{Ca}^{2+}$ sensor subunit of the calcium-release-activated calcium (CRAC) channel on ER. It is believed to gather into puncta near the cell surface, forming higher-order complexes with Orai1, which is integrated in the plasma membrane, when the $\mathrm{Ca}^{2+}$ store is depleted. Jurkat $\mathrm{T}$ cells cultured on ASEM dish were treated with and without thapsigargin [2]. The cells are then fixed and perforated. Labeled with immuno-gold, a dynamic string-like gathering of STIM1 on the ER was visualized in response to $\mathrm{Ca}^{2+}$ store depletion using ASEM (Figure 2).

The throughput of immuno-ASEM is comparable with that of immuno-OM, because open structure of ASEM dish allows efficient labeling and washing (medium change). Immune-ASEM visualized microtubule and F-actin in the axons and synapses of neuron primary culture [2], CD44 receptor in glioma cell [3], and leg molecular complexes of mycoplasma [4]. The ASEM system was applied to observe nuclei in the surface of tissues, which could be applied to intra-operative cancer diagnosis [1]. ASEM also visualized non-stained protein micro crystals in solution [5]. It monitored non-biological dynamic phenomena, i.e., salt crystal formation, self-organization of particles, electrochemical deposition, and the changes occurring during the solid / liquid phase transition of solder [6]. 
References:

[1] H. Nishiyama et al., J Struct Biol 169, (2010) 438-449.

[2] Y. Maruyama et al., J Struct Biol 180, (2012) 259-270.

[3] T. Murai et al., J. Biol. Chem. 286 (2011) 1999-2007.

[4] C. Sato et al., Biochem. Biophys. Res. Commun. 417, (2012) 1213-1218.

[5] Y. Maruyama et al., Int. J. Mol. Sci. 13, (2012) 10553-10567.

[6] M. Suga et al., Ultramicroscopy 111, (2011)1650-1658.

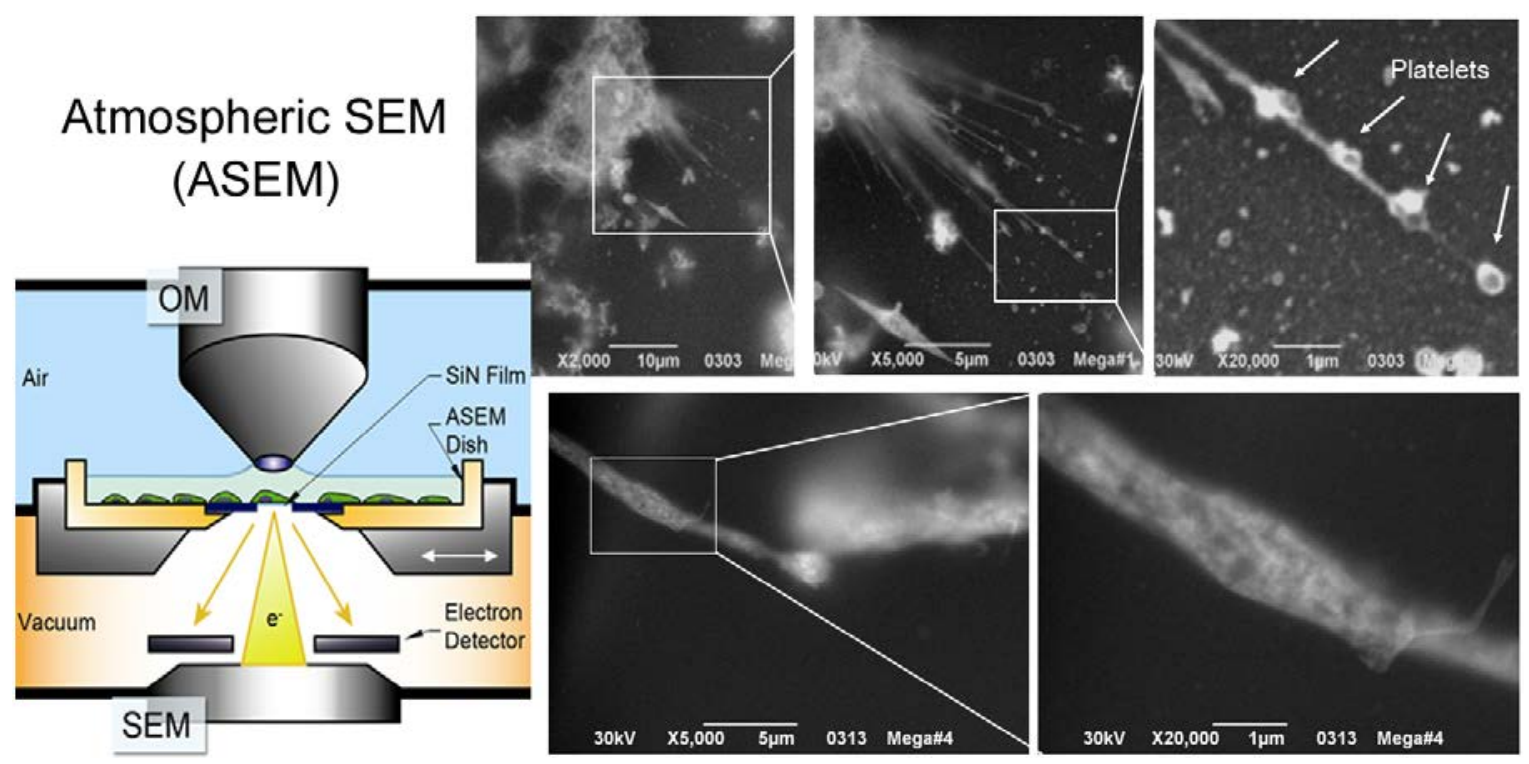

Figure 1. Configuration of the ASEM and the moment of platelet generation from MKs. The SEM has a totally inverted structure, with electron gun at the bottom. MKs cultured on the electron transparent film of ASEM dish were fixed, stained with Ti-blue (upper) or phosphotungstic acid (lower), and observed using ASEM. The cells extended pseudopodia of beaded strings including vesicles.

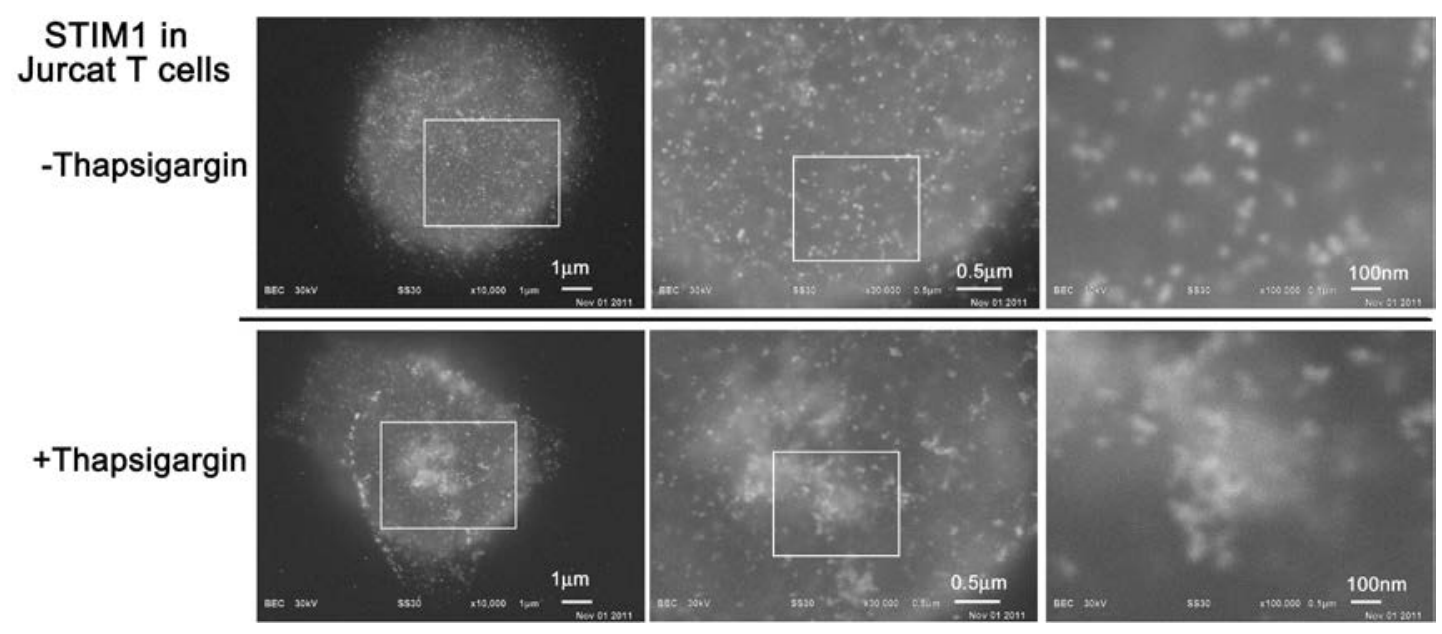

Figure 2. Rearrangement of STIM1 in response to $\mathrm{Ca}^{2+}$ store depletion of ER in Jurkat T cells. T cells were treated with and without thapsigargin, fixed and perforated. STIM1 was labeled with FluoroNanogold, gold-enhanced and observed using ASEM. (Upper) Without store depletion. STIM1 was distributed on ER. (Lower) With store depletion. STIM1 proteins gathered, forming strings on ER. 